\title{
BIODIVERSIDAD AGRICOLA: VARIEDADES DE ALMENDRO Y OLIVO DE SECANO DEL SURESTE IBÉRICO
}

\section{Antonio R. Ricarte Sabater}

CIBIO, Universidad de Alicante

\section{INTRODUCCION}

El conocimiento de las variedades tradicionales utilizadas en los cultivos agrícolas de almendros, Prunus dulcis (Mill.) D. A. Webb (Rosaceae), y olivos, Olea europaea L. (Oleaceae), permite disponer de una información más amplia y concreta sobre su uso en tierras de secano. El hallazgo de localismos, conocidos únicamente en pequeñas áreas, da una idea del potencial genético guardado en los cultivos del Sureste Ibérico. A su vez, nos hace conscientes de la necesidad de conservar esta parte de la biodiversidad, que son las variedades de plantas cultivadas.

El objetivo de este artículo es poner de relieve el valor etnobotánico de los cultivos tradicionales de almendro y olivo, en unos tiempos en los que se conservan sólo los restos de una agricultura tradicional, antiguamente mucho más importante, por ser el principal sostén y mantenimiento de sus pobladores.

Hoy día, la agricultura tradicional está relegada a un segundo plano debido a que los terrenos desti- nados a ella están siendo sustituidos por urbanizaciones y zonas industriales, o por su abandono al dar un rendimiento económico insuficiente. A pesar de esta disminución de la actividad agrícola, muchos agricultores aún conservan, como actividad complementaria y/o secundaria, pequeñas propiedades que siguen cultivando y que los abastecen de almendra, aceituna, y también de frutales, uva de mesa y cítricos, que destinan, preferentemente, al autoconsumo. De todas las partidas rurales presentes en el municipio alicantino de Sant Vicent del Raspeig, únicamente la denominada "Raspeig" conserva en parte esta actividad tradicional, protegida por el Plan General de Ordenación Urbana (según información disponible en el año 2002).

No se conocen estudios específicos sobre la etnoflora de Sant Vicent, pero ya el naturalista Cavanilles, en el S. XVIII, cita la bondad al paladar del aceite y la gran cantidad de almendras producidas en los campos de Alicante, en los que se incluían para aquel entonces los territorios actuales de Sant Vicent. Adicionalmente, cita la resistencia de los cultivos de esta región a las grandes pérdidas 


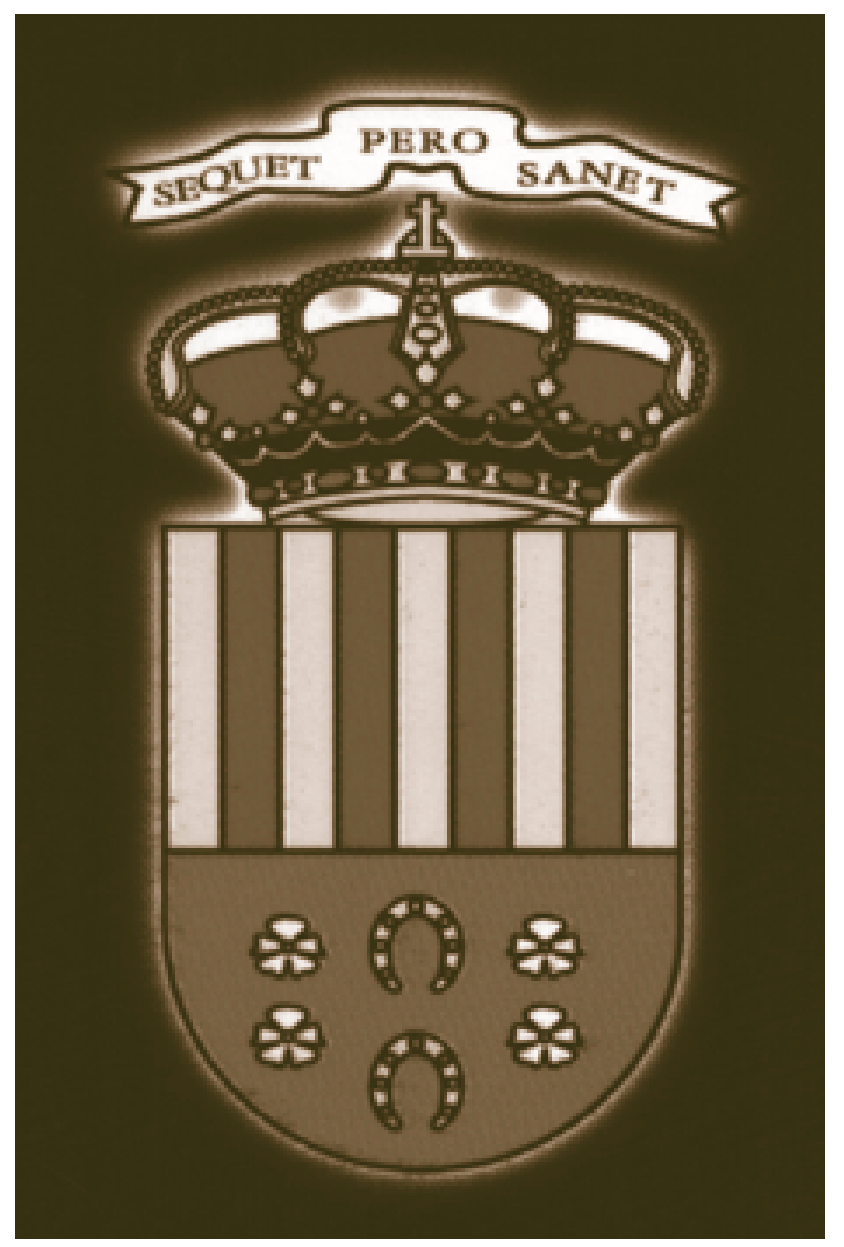

Figura 1. Escudo de Sant Vicent del Raspeig.

agrícolas de 1792, que afectaron a todo el Reino de Valencia. Esta resistencia fue probablemente debida a la benevolencia del clima del lugar a pesar de su sequedad. Muchas de aquellas pérdidas tuvieron lugar debido a fuertes heladas que sacudieron los alrededores de la provincia.

En cuanto a variedades concretas, Cavanilles cita la oliva "Grosal" (no confundir con la "Gordal") como la preferida por los antiguos agricultores de estos campos. Aún hoy, más de 200 años después, existen pies "Grosales" en el término de Sant Vicent.

La relevancia del almendro en el secano de Sant Vicent queda reflejada en su propio escudo, el cual cuenta con 4 flores de almendro junto con 2 herraduras (Figura 1), por ser la actividad agrícola ligada a este cultivo y la profesión de carretero los pilares de esta sociedad hasta la llegada de las nuevas tendencias, más propias del área de la industria y los servicios.
Los usos de las almendras son varios, desde la elaboración de productos típicos navideños (turrón, etc.) hasta condimento en comidas, pasando por consumo directo de la semilla, tostadas o no. En cualquier caso, se trata, frecuentemente, de usos asociados a tradiciones y de un ámbito más o menos local a pesar de la promoción del turrón fuera de las fronteras nacionales españolas o del uso generalizado en repostería.

En cuanto a los olivos, hay que decir que el territorio de Sant Vicent se ubica dentro de la zona olivarera séptima: Levante (según la clasificación de zonas olivareras españolas atendiendo a las características productivas, del Ministerio de Agricultura, 1972) que abarca las provincias de Murcia, Alicante y Valencia. En ella existe un amplio mosaico de variedades. Los principales usos de las aceitunas son la elaboración de aceite común y, en un plano secundario pero igualmente importante, el aderezo, que recoge diferentes modalidades tradicionales o no, y más o menos locales.

\section{EL ÁREA DE ESTUDIO}

El municipio de Sant Vicent del Raspeig (Alicante, España), dista tan sólo $6 \mathrm{~km}$ de Alicante. El terreno que ocupa es muy accidentado, descendiendo progresivamente desde la Serra del Ventós al norte, pasando por la Lloma Redona (250 metros sobre el nivel del mar), hasta alcanzar la llanura (109 $\mathrm{m}$ de altitud). La superficie total del término asciende a 3.934 hectáreas, de las cuales unas 370 son espacios libres rústicos de protección. El clima de la población es suave y seco. Los valores medios de temperatura oscilan entre los $11^{\circ} \mathrm{C}$ de enero y los $25^{\circ}$ de julio y agosto, mientras que las precipitaciones no superan por lo general los $340 \mathrm{~mm}$ anuales.

La recopilación de datos se hizo a través de encuentros con diferentes particulares (8 en total) que respondieron a la petición del autor y cedieron muestras de tallo, hojas y frutos del mayor número posible de las variedades investigadas.

De las 5 partidas rurales que comprende el municipio, la recopilación de información de carácter émico y la toma de muestras se desarrollaron en la partida Raspeig y, en menor medida, en las partidas Canastell y Boqueres. 
En lo que se refiere al análisis de los materiales recolectados, se procedió a la conservación de las almendras, semillas de las aceitunas y partes somáticas de los vegetales, y se realizó una caracterización de almendras y aceitunas, siendo Felipe (2000) y Barranco et al (1997) las obras tomadas como referencia, respectivamente.

\section{DESCRIPCIÓN DE LAS VARIEDADES}

A continuación se detalla la ubicación taxonómica de las diferentes variedades desde el punto de vista botánico y etnobotánico según Ribera et al (1997). Las tablas 1 y 2 resumen la caracterización de sus frutos.

Familia Rosaceae

Subfamilia Prunoideae

Género Prunus

1: Almendro dulce común (Prunus dulcis (Mill.) D. A. Webb var. dulcis)

* Almendros dulces tempranos (florecen en enero y primera quincena de febrero):

Almendra Castellet (Prunus dulcis etnovar. "Castellet"

Almendra Pestañeta (Prunus dulcis etnovar. "Pestañeta")

Almendra Planeta (Prunus dulcis etnovar. "Planeta")

* Almendros dulces intermedios. Florecen a mediados de febrero.

Almendra Comuna (Prunus dulcis etnovar. "Comuna")

Almendra Marcona (Prunus dulcis cvar. 'Marcona')

* Almendros dulces tardíos. Florecen a partir de mediados del mes de febrero.

Almendra Desmayo (Prunus dulcis etnovar. "Desmayo")

Almendra Guara (Prunus dulcis cvar. 'Guara')

2: Almendro mollar (Prunus dulcis (Miller) D. A. Webb var. fragilis Buchheim)

3: Almendro amargo (Prunus dulcis (Miller) D. A. Webb var. amara (DC) Buchheim

Clasificación según dureza de la cáscara de las variedades inéditas (no se hallan referenciadas en la bibliografía consultada):
Dura:

Almendra Dura Agostera

Almendra Rompesacos

Almendra Tocha Americana

Almendra Tou

Almendra Balsamera

Blanda:

Almendra Formigueta Grande

Almendra Piquet

Intermedia:

Almendra Elchera

Almendra Soguera (Foto 1)

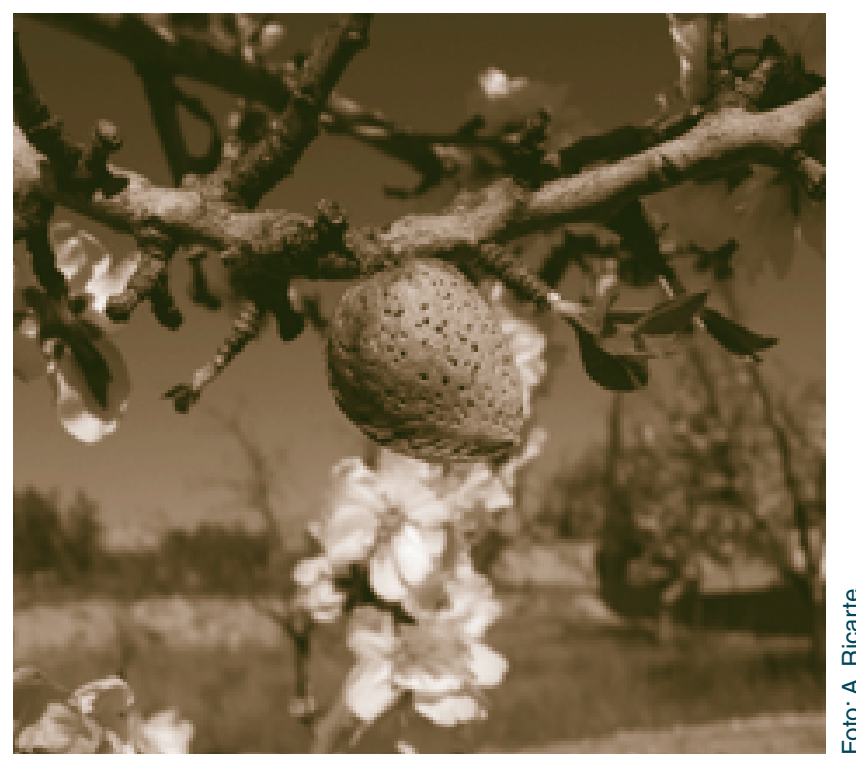

Foto 1. Almendra de la variedad "Soguera" sobre árbol en flor.

Familia Oleaceae

Género Olea

1: Olivo común (Olea europaea L.)

Oliva Cornicabra (Olea europaea var. europaea etnovar. "Cornicabra")

Oliva Gordal (Olea europaea var. europaea etnovar. "Gordal")

Oliva Manzanilla de Andalucía (Olea europaea ssp europaea cvar. 'Manzanilla')

Oliva Picual (Olea europaea var. europaea etnovar. "Picual")

Oliva Xanglot Real (Olea europaea var. europaea etnovar. "Xanglot Real") 
Tabla 1 Caracterización de las almendras con cáscara pertenecientes a las variedades inéditas.

\begin{tabular}{|c|c|c|c|c|c|c|c|}
\hline \multirow[t]{2}{*}{$\begin{array}{l}\text { NOMBRE DE LA } \\
\text { VARIEDAD }\end{array}$} & \multicolumn{2}{|c|}{$\begin{array}{c}\text { TAMAÑO } \\
\text { (media } \pm \\
\text { desviación estándar) }\end{array}$} & \multirow[t]{2}{*}{ FORMA } & \multirow[t]{2}{*}{$\begin{array}{c}\text { ASPECTO } \\
\text { EXTERNO DEL } \\
\text { ENDOCARPO }\end{array}$} & \multirow[t]{2}{*}{$\begin{array}{c}\text { SUTURA } \\
\text { VENTRAL }\end{array}$} & \multirow[t]{2}{*}{ APÍCULO } & \multirow[t]{2}{*}{ OBSERVACIONES } \\
\hline & $\begin{array}{l}\text { Largo }(\mathrm{mm}) \\
\end{array}$ & Ancho (mm) & & & & & \\
\hline Guara & $\begin{array}{c}30.6 \\
( \pm 0.177)\end{array}$ & $\begin{array}{c}21.44 \\
( \pm 0.339)\end{array}$ & Amigdaloide & $\begin{array}{l}\text { Medianamente } \\
\text { Punteado }\end{array}$ & $\begin{array}{l}\text { Moderada-mente } \\
\text { resaltada }\end{array}$ & $\begin{array}{c}\text { Poco } \\
\text { pronunciado }\end{array}$ & \\
\hline Dura Agostera & 29.75 & 21.81 & $\begin{array}{c}\text { Elíptica- } \\
\text { acorazonada }\end{array}$ & Poco punteado & $\begin{array}{l}\text { Muy resaltada (en } \\
\text { las cercanías del } \\
\text { apículo) }\end{array}$ & Poco pronunciado & \\
\hline Rompesacos & $\begin{array}{c}40.96 \\
( \pm 2.192)\end{array}$ & $\begin{array}{c}24.32 \\
( \pm 3.84)\end{array}$ & Larga & $\begin{array}{l}\text { Medianamente } \\
\text { punteado y } \\
\text { ligeramente } \\
\text { asurcado }\end{array}$ & Poco resaltada & Poco pronunciado & $\begin{array}{l}\text { Aspecto } \\
\text { "muy rústico" }\end{array}$ \\
\hline Tocha Americana & $\begin{array}{c}30.73 \\
( \pm 1.098)\end{array}$ & $\begin{array}{c}19.12 \\
( \pm 0.231)\end{array}$ & $\begin{array}{l}\text { Elíptica- } \\
\text { alargada }\end{array}$ & $\begin{array}{l}\text { Medianamente } \\
\text { Punteado }\end{array}$ & $\begin{array}{l}\text { Moderadamente } \\
\text { resaltada }\end{array}$ & $\begin{array}{l}\text { Moderadamente } \\
\text { pronunciado }\end{array}$ & \\
\hline Tou & $\begin{array}{l}29.78 \\
( \pm 2.352)\end{array}$ & $\begin{array}{c}19.78 \\
( \pm 1.082)\end{array}$ & $\begin{array}{c}\text { Elíptica- } \\
\text { amigdaloide }\end{array}$ & $\begin{array}{l}\text { Medianamente } \\
\text { Punteado }\end{array}$ & $\begin{array}{l}\text { Moderadamente } \\
\text { resaltada }\end{array}$ & $\begin{array}{l}\text { Moderadamente } \\
\text { pronunciado } \\
\text { (pero bastante } \\
\text { punzante) }\end{array}$ & \\
\hline Balsamera & $\begin{array}{c}30.1 \\
( \pm 0.233)\end{array}$ & $\begin{array}{c}22.53 \\
( \pm 0.021)\end{array}$ & $\begin{array}{l}\text { Acorazonada } \\
\text {-elíptica }\end{array}$ & $\begin{array}{l}\text { Medianamente } \\
\text { Punteado }\end{array}$ & $\begin{array}{l}\text { Moderadamente } \\
\text { resaltada }\end{array}$ & Poco pronunciado & \\
\hline Formigueta Grande & $\begin{array}{c}34.72 \\
( \pm 2.454)\end{array}$ & $\begin{array}{c}25.41 \\
( \pm 1.909)\end{array}$ & $\begin{array}{l}\text { Redonda- } \\
\text { Acorazonada }\end{array}$ & $\begin{array}{l}\text { Poco punteado } \\
\text { y ligeramente } \\
\text { Asurcado }\end{array}$ & $\begin{array}{l}\text { Moderadamente } \\
\text { resaltada }\end{array}$ & $\begin{array}{l}\text { Muy pronunciado } \\
\text { (poco punzante) }\end{array}$ & $\begin{array}{l}\text { Aspecto } \\
\text { "rústico" }\end{array}$ \\
\hline Piquet & $\begin{array}{c}33.43 \\
( \pm 0.445)\end{array}$ & $\begin{array}{c}19.82 \\
( \pm 0.085)\end{array}$ & Larga & $\begin{array}{l}\text { Medianamente } \\
\text { Punteado }\end{array}$ & Poco resaltada & $\begin{array}{l}\text { Muy pronunciado } \\
\text { (punzante) }\end{array}$ & $\begin{array}{c}\text { Zona apicular } \\
\text { doblada }\end{array}$ \\
\hline Elchera & - & - & - & - & - & - & - \\
\hline Soguera & 32.91 & 26.58 & $\begin{array}{l}\text { Acorazonada } \\
\text {-elíptica }\end{array}$ & $\begin{array}{l}\text { Poco punteado } \\
\text { y ligeramente } \\
\text { asurcado }\end{array}$ & $\begin{array}{l}\text { Moderadamente } \\
\text { resaltada }\end{array}$ & $\begin{array}{l}\text { Moderadamente } \\
\text { pronunciado }\end{array}$ & $\begin{array}{l}\text { Aspecto } \\
\text { "rústico" }\end{array}$ \\
\hline
\end{tabular}

\section{2: Olivos occidentales, primitivos y silvestres} (Olea maroccana Greuter y Burdet)

Oliva Blanqueta (Olea maroccana etnovar. "Blanqueta")

Oliva del Cuquello (Olea maroccana etnovar. "Cuquello")

Variedades inéditas:

\section{Oliva Callosina (Foto 2) \\ Oliva Grosal \\ Oliva Hermana}

\section{DISCUSIÓN}

De las 26 variedades de olivo y almendro registradas en el trabajo, 9 de almendro y 3 de olivo (incluyendo la oliva "Grosal", que sólo está referenciada por Cavanilles) son inéditas, lo que da una idea del potencial etnobotánico desconocido de los campos de cultivo de secano relictos de Sant Vicent del Raspeig. Ello es una prueba más a favor de la

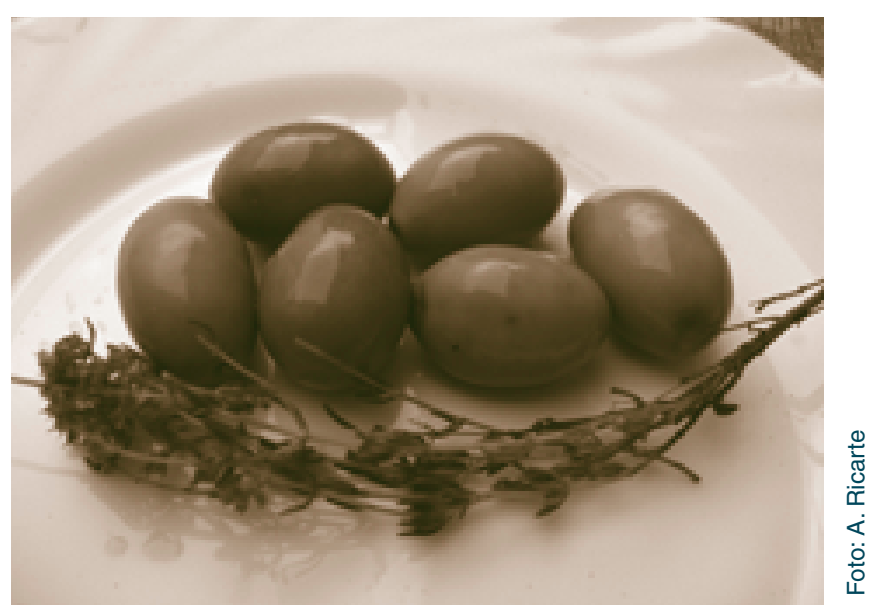

Foto 2. Aceitunas de la variedad "Callosina" adobadas con agua, sal, pebrella y tomillo.

conservación del acervo genético de estos núcleos agrícolas que, en un momento dado, pueden ser fuente de variedades con características deseables para su cultivo (Felipe, 2000), entre las cuales se podría citar la resistencia frente a determinadas plagas. Respecto a ello, es de destacar la referencia que 


\begin{tabular}{|c|c|c|c|c|c|}
\hline \multirow{2}{*}{$\begin{array}{l}\text { NOMBRE } \\
\text { DE LA } \\
\text { VARIEDAD }\end{array}$} & \multicolumn{2}{|c|}{$\begin{array}{c}\text { TAMAÑO } \\
\text { (media } \pm \\
\text { desviación estándar) }\end{array}$} & \multirow{2}{*}{ FORMA } & \multirow{2}{*}{ COLOR } & \multirow{2}{*}{$\begin{array}{c}\text { FORMA } \\
\text { ENDOCARPO }\end{array}$} \\
\hline & $\begin{array}{l}\text { Largo } \\
(\mathrm{mm})\end{array}$ & $\begin{array}{l}\text { Ancho } \\
(\mathrm{mm})\end{array}$ & & & \\
\hline Callosina & $\begin{array}{c}27.36 \\
( \pm 1.426)\end{array}$ & $\begin{array}{c}18.83 \\
( \pm 0.472)\end{array}$ & Elíptica & Pruinosa (un poco moteada) & Estriado; apiculado \\
\hline Grosal & $\begin{array}{c}29.54 \\
( \pm 0.714)\end{array}$ & $\begin{array}{c}20.44 \\
( \pm 1.047)\end{array}$ & Elíptica & $\begin{array}{c}\text { Pruinosa/Variegada/Moteada } \\
\text { (motas pequeñas pero muy patentes) }\end{array}$ & Estriado; apiculado \\
\hline Hermana & $\begin{array}{c}20.38 \\
( \pm 2.12)\end{array}$ & $\begin{array}{c}14.14 \\
( \pm 0.953)\end{array}$ & Elíptica & Pruinosa/Variegada (un poco moteada) & Estriado; apiculado \\
\hline
\end{tabular}

Tabla 2. Caracterización de las aceitunas pertenecientes a las variedades oleícolas inéditas.

uno de los informantes hizo a una variedad de olivo que nunca era atacada por la "mosca del olivo" ni captaba otras enfermedades; además se trataba de una oliva con altos rendimientos y, por tanto, también valiosa desde el punto de vista productivo. No obstante, se desconoce el nombre de esta variedad y su localización en las tierras del municipio es indeterminada.

Además, la biodiversidad etnobotánica de los campos de Sant Vicent se halla, en muchos casos, altamente concentrada. Por ejemplo, en una parcela de $2.400 \mathrm{~m}^{2}$ se pudieron recolectar muestras de 6 variedades de almendro, aunque existían otras, entre las cuales destacaban unos pies poco desarrollados con características de almendro "borde o amargo" pero, curiosamente, de almendra dulce, no amarga, perteneciente a una variedad de nombre también desconocido.

Gracias al estatus de especial protección agrícola asignado a la partida Raspeig, se podrá conservar toda esta diversidad varietal, producto, en muchos casos, de siglos de evolución, en gran medida dirigida por el hombre. Aunque este panorama puede parecer algo alentador, no hay que olvidar que estos territorios del Raspeig son de especial protección agrícola, en buena medida, gracias a la existencia de grandes superficies de cultivo extensivo, tuteladas por empresas agrícolas. Los resultados muestran la existencia de núcleos agrícolas tradicionales, muy valiosos desde el punto de vista etnobotánico y agronómico, en la partida Raspeig y añadir éste como argumento de peso en esa parte del ordenamiento territorial que asigna especial relevancia agrícola a esta partida. Sin embargo, es necesario la realización de trabajos etnobotánicos de mayor profundidad que resalten la importancia de los campos de secano del Sureste Ibérico.

En cuanto a las variedades en concreto, son de destacar la variedad "Grosal” y "Callosina" de olivo en estos terrenos, que gozan de un rango especial en las tradiciones agrícolas de los trabajadores del campo. A su vez, resaltamos la elaboración de olivas "marsías", como una costumbre arraigada y bastante específica de esta localidad, gracias a la cual se mantienen ciertos pies de olivo, con el único fin de elaborarlas. Dichas olivas "marsías" podrían pasar a promocionarse como elemento plenamente constitutivo de la gastronomía local, enriqueciéndola así y estimulando la atracción de "turistas de mesa". Lo mismo se podría decir en el caso de las olivas aderezadas al estilo tradicional, con sal, agua e hinojo (Foeniculum vulgare Miller) (o tomillo con pebrella, Thymus vulgaris L. y Thymus piperella L., respectivamente) (Foto 2 ).

Tanto en el caso del cultivo del olivo como del almendro, existe una elevada permeabilidad a las tendencias externas, y la entrada de variedades procedentes de otras regiones de España (por ejemplo, "Manzanilla de Andalucía" o "Tocha Americana"). Este efecto se ve acentuado por los procesos de selección acelerada a los que hoy día están sometidos los cultivos, gracias al desarrollo tecnológico. Una entrada de nuevas variedades que suponga la desaparición de las locales es un fenómeno que afecta negativamente esta biodiversidad, pero una entrada controlada y la tolerancia de la coexistencia entre las variedades autóctonas y alóctonas tendría un 
efecto positivo desde el punto de vista de la diversidad genética.

Es un hecho peculiar que el producto de ciertos almendros ("Mollar") se destine al autoconsumo. Todo ello gracias a ciertos rasgos que hacen a la almendra especialmente apetecible y fácil de consumir (cáscara blanda, por ejemplo). Lo mismo ocurre con la elaboración de olivas aderezadas, muy ligado a la tradición, a pesar de que van desapareciendo las fuentes de tales productos: las distintas variedades de olivo y almendro.

Hasta hoy, los agricultores locales se muestran como la principal fuente informativa, ya que éstos son los que más directamente experimentan el cultivo de los distintos árboles. Esto supone que, con la pérdida de estas formas de vida tradicionales, también se pierda todo el conocimiento acumulado, sobre el cultivo del olivo y del almendro y, finalmente, también parte de la biodiversidad agrícola más próxima a nosotros.

\section{AGRADECIMIENTOS}

Agradecer a todos los informantes, sin los cuales este trabajo no hubiera sido posible; y a Segundo Ríos Ruíz, profesor del CIBIO que tuteló el desarrollo del mismo.

\section{REFERENCIAS BIBLIOGRÁFICAS}

BARRANCO, D.; FERNÁNDEZ-ESCOBAR, R.; RALLO, L. 1998. El Cultivo del Olivo. 2a edición revisada y ampliada. Coedición Junta de Andalucía (Consejería de Agricultura y Pesca) y ediciones Mundi-Prensa, 661pp.

CAVANILLES, A. J., 1797. Observaciones sobre la Historia Natural, geografía, agricultura, población y frutos del Reyno de Valencia. Impreso por la Imprenta Real, siendo regente Don Pedro Julián Pereyra, impresor de cámara de S.M. Reeditado en la actualidad por: Bancaja (Obra Social), con el título de "Las Observaciones de Cavanilles. Doscientos años después"; y autores: Lacarra, J., Sánchez, X. y Jarque, F. 1997; 512 pp.

FELIPE, A. J. 2000. El Almendro; Serie: El Material Vegetal, I. Ed. Integrum, 461 pp.

RIVERA NÚNEEZ, D.; OBÓN DE CASTRO, C.; RÍOS RUÍZ, S.; SELMA FERRÁNDEZ, C.; MÉNDEZ COLMENERO, F.; VERDE LÓPEZ, A. y CANO TRIGUEROS, F. 1997. Las variedades tradicionales de frutales de la Cuenca del Río Segura. Catálogo etnobotánico (1): frutos secos, oleaginosos, frutales de hueso, almendros y frutales de pepita. Servicio de publicaciones de la Universidad de Murcia, 360 pp. 